\title{
Iterative algorithm for a family of split equilibrium problems and fixed point problems in Hilbert spaces with applications
}

Shenghua Wang ${ }^{1}$, Xiaoying Gong ${ }^{2}$, Afrah AN Abdou ${ }^{3}$ and Yeol Je Cho ${ }^{4,5 *}$

\author{
Correspondence: yjcho@gnu.ac.kr \\ ${ }^{4}$ Department of Mathematics \\ Education and RINS, Gyeongsang \\ National University, Jinju, 660701 \\ Korea \\ ${ }^{5}$ Center for General Education, \\ China Medical University, Taichung, \\ 40402, Taiwan \\ Full list of author information is \\ available at the end of the article
}

\begin{abstract}
In this paper, we propose an iterative algorithm and, by using the proposed algorithm, prove some strong convergence theorems for finding a common element of the set of solutions of a finite family of split equilibrium problems and the set of common fixed points of a countable family of nonexpansive mappings in Hilbert spaces. An example is given to illustrate the main result of this paper. As an application, we construct an algorithm to solve an optimization problem.
\end{abstract}

MSC: 47H10; 54H25;65J15; $90 \mathrm{C} 30$

Keywords: split equilibrium problem; nonexpansive mapping; split feasible solution problem; Hilbert space

\section{Introduction}

Throughout this paper, let $\mathbb{R}$ denote the set of all real numbers, $\mathbb{N}$ denote the set of all positive integer numbers, $H$ be a real Hilbert space and $C$ be a nonempty closed convex subset of $H$. A mapping $S: C \rightarrow C$ is said to nonexpansive if

$$
\|S x-S y\| \leq\|x-y\|
$$

for all $x, y \in C$. The set of fixed points of $S$ is denoted by Fix $(S)$. It is known that the set $\operatorname{Fix}(S)$ is closed and convex.

Let $F: C \times C \rightarrow \mathbb{R}$ be a bifunction. The equilibrium problem for $F$ is to find $z \in C$ such that

$$
F(z, y) \geq 0
$$

for all $y \in C$. The set of all solutions of the problem (1.1) is denoted by $\operatorname{EP}(F)$, i.e.,

$$
\operatorname{EP}(F)=\{z \in C: F(z, y) \geq 0, \forall y \in C\} .
$$

From the problem (1.1), we can consider some related problems, that is, variational inequality problems, complementarity problems, fixed point problems, game theory and (http://creativecommons.org/licenses/by/4.0/), which permits unrestricted use, distribution, and reproduction in any medium, provided you give appropriate credit to the original author(s) and the source, provide a link to the Creative Commons license, and indicate if changes were made. 
other problems. Also, many problems in physics, optimization, and economics can be reduced to finding a solution of the problem (1.1) (see [1-4]).

In 1997, Combettes and Hirstoaga [5] introduced an iterative scheme of finding a solution of the problem (1.1) under the assumption that $\mathrm{EP}(F)$ is nonempty. Later on, many iterative algorithms were considered to find a common element of the set of $\operatorname{Fix}(S) \cap \operatorname{EP}(F)$ (see [6-11]).

Recently, some new problems called split variational inequality problems were considered by some authors. Especially, Censor et al. [12] initially studied this class of split variational inequality problems.

Let $H_{1}$ and $H_{2}$ be two real Hilbert spaces. Given the operators $f: H_{1} \rightarrow H_{1}$ and $g: H_{2} \rightarrow$ $H_{2}$, bounded linear operator $A: H_{1} \rightarrow H_{2}$, and nonempty closed convex subsets $C \subset H_{1}$ and $Q \subset H_{2}$, the split variational inequality problem is formulated as follows:

Find a point $x^{*} \in C$ such that

$$
\left\langle f\left(x^{*}\right), x-x^{*}\right\rangle \geq 0
$$

for all $x \in C$ and such that

$$
y^{*}=A x^{*} \in Q \quad \text { solves } \quad\left\langle g\left(y^{*}\right), y-y^{*}\right\rangle \geq 0
$$

for all $y \in Q$.

After investigating the algorithm of Censor et al., Moudafi [13] introduced a new iterative scheme to solve the following split monotone variational inclusion:

Find $x^{*} \in H_{1}$ such that

$$
0 \in f\left(x^{*}\right)+B_{1}\left(x^{*}\right)
$$

and such that

$$
y^{*}=A x^{*} \in H_{2} \quad \text { solves } \quad 0 \in g\left(y^{*}\right)+B_{2}\left(y^{*}\right)
$$

where $B_{1}: H_{i} \rightarrow 2^{H_{i}}$ is a set-valued mappings for $i=1,2$.

In 2013, Kazmi and Rizvi [14] considered a new class of split equilibrium problems. Let $F_{1}: C \times C \rightarrow \mathbb{R}$ and $F_{2}: Q \times Q \rightarrow \mathbb{R}$ be two bifunctions and $A: H_{1} \rightarrow H_{2}$ be a bounded linear operator. The split equilibrium problem is as follows:

Find $x^{*} \in C$ such that

$$
F_{1}\left(x^{*}, x\right) \geq 0
$$

for all $x \in C$ and such that

$$
y^{*}=A x^{*} \in Q \quad \text { solves } \quad F_{2}\left(y^{*}, y\right) \geq 0
$$

for all $y \in Q$. The set of all solutions of the problems (1.2) and (1.3) is denoted by $\Omega$, i.e.,

$$
\Omega=\left\{z \in C: z \in \mathrm{EP}\left(F_{1}\right) \text { such that } A z \in \operatorname{EP}\left(F_{2}\right)\right\} .
$$


For more details as regards the split equilibrium problems, refer to $[15,16]$, in which the author gave an iterative algorithm to find a common element of the sets of solutions of the split equilibrium problem and hierarchical fixed point problem.

In this paper, inspired by the results in [14] and [16], we propose an iterative algorithm to find a common element of the set of solutions for a family of split equilibrium problems and the set of common fixed points of a countable family of nonexpansive mappings. In particular, we use some new methods to prove the main result of this paper. As an application, we propose an iterative algorithm to solve a split variational inequality problem.

\section{Preliminaries}

Let $H$ be a Hilbert space and $C$ be a nonempty closed subset of $H$. For each point $x \in H$, there exists a unique nearest point of $C$, denoted by $P_{C} x$, such that

$$
\left\|x-P_{C} x\right\| \leq\|x-y\|
$$

for all $y \in C$. Such a $P_{C}$ is called the metric projection from $H$ onto $C$. It is well known that $P_{C}$ is a firmly nonexpansive mapping from $H$ onto $C$, i.e.,

$$
\left\|P_{C} x-P_{C} y\right\|^{2} \leq\left\langle P_{C} x-P_{C} y, x-y\right\rangle
$$

for all $x, y \in H$. Further, for any $x \in H$ and $z \in C, z=P_{C} x$ if and only if

$$
\langle x-z, z-y\rangle \geq 0
$$

for all $y \in C$.

A mapping $B: C \rightarrow H$ is called $\alpha$-inverse strongly monotone if there exists $\alpha>0$ such that

$$
\langle x-y, B x-B y\rangle \geq \alpha\|B x-B y\|^{2}
$$

for all $x, y \in H$. For each $\lambda \in(0,2 \alpha], I-\lambda B$ is a nonexpansive mapping of $C$ into $H$ (see [17]).

Consider the following variational inequality for an inverse strongly monotone mapping $B$ :

Find $u \in C$ such that

$$
\langle v-u, B u\rangle \geq 0
$$

for all $v \in C$. The set of solutions of the variational inequality is denoted $\operatorname{VI}(C, B)$. It is well known that

$$
u \in \mathrm{VI}(C, B) \quad \Longleftrightarrow u=P_{C}(u-\lambda B u)
$$

for any $\lambda>0$. By this property, we can use a simple method to show that $u \in \operatorname{VI}(C, B)$. In fact, let $\left\{x_{n}\right\}$ be a sequence in $C$ with $x_{n} \rightarrow u$. If $x_{n}-P_{C}(I-\lambda B) x_{n} \rightarrow 0$, then, by the demiclosedness principle, it follows that $u=P_{C}(I-\lambda B)$, i.e., $u \in \operatorname{VI}(C, B)$. In Section 3, we use this method to show the conclusions of our main results in this paper. 
Let $S: C \rightarrow C$ be a mapping. It is well known that $S$ is nonexpansive if and only if the complement $I-S$ is $\frac{1}{2}$-inverse strongly monotone (see [18]). Assume that $\operatorname{Fix}(S) \neq \emptyset$. Then we have

$$
\|S x-x\|^{2} \leq 2\langle x-S x, x-\hat{x}\rangle
$$

for all $x \in C$ and $\hat{x} \in \operatorname{Fix}(S)$, which is obtained directly from

$$
\begin{aligned}
\|x-\hat{x}\|^{2} & \geq\|S x-S \hat{x}\|^{2}=\|S x-\hat{x}\|^{2}=\|S x-x+(x-\hat{x})\|^{2} \\
& =\|S x-x\|^{2}+\|x-\hat{x}\|^{2}+2\langle S x-x, x-\hat{x}\rangle .
\end{aligned}
$$

Let $F$ be a bifunction of $C \times C$ into $\mathbb{R}$ satisfying the following conditions:

(A1) $F(x, x)=0$ for all $x \in C$;

(A2) $F$ is monotone, i.e., $F(x, y)+F(y, x) \leq 0$ for all $x, y \in C$;

(A3) for each $x, y, z \in C, \lim _{t \downarrow 0} F(t z+(1-t) x, y) \leq F(x, y)$;

(A4) for each $x \in C, y \mapsto F(x, y)$ is convex and lower semi-continuous.

Lemma 2.1 [19] Let $C$ be a nonempty closed convex subset of a Hilbert space $H$ and $F$ : $C \times C \rightarrow \mathbb{R}$ be a bifunction which satisfies the conditions (A1)-(A4). For any $x \in H$ and $r>0$, define a mapping $T_{r}: H \rightarrow C$ by

$$
T_{r}^{F}(x)=\left\{z \in C: F(z, y)+\frac{1}{r}\langle y-z, z-x\rangle \geq 0, \forall y \in C\right\} .
$$

Then $T_{r}^{F}$ is well defined and the following hold:

(1) $T_{r}^{F}$ is single-valued;

(2) $T_{r}^{F}$ is firmly nonexpansive, i.e., for any $x, y \in H$,

$$
\left\|T_{r}^{F} x-T_{r}^{F} y\right\|^{2} \leq\left\langle T_{r}^{F} x-T_{r}^{F} y, x-y\right\rangle
$$

(3) $\operatorname{Fix}\left(T_{r}^{F}\right)=\mathrm{EP}(F)$;

(4) $\mathrm{EP}(F)$ is closed and convex.

Lemma 2.2 [20] Let $F: C \times C \rightarrow \mathbb{R}$ be a bifunction satisfying the conditions (A1)-A(4). Let $T_{r}^{F}$ and $T_{s}^{F}$ be defined as in Lemma 2.1 with $r, s>0$. Then, for any $x, y \in H$, one has

$$
\left\|T_{r}^{F} x-T_{s}^{F} y\right\| \leq|x-y|+\left|1-\frac{s}{r}\right|\left\|T_{r}^{F} x-x\right\| .
$$

Remark 2.1 In [20], some other conditions are required besides the conditions (A1)-(A4). In fact, the conditions (A1)-(A4) are enough for Lemma 2.2. For the proof, refer to $[9,20]$.

Lemma 2.3 [9] Let $F: C \times C \rightarrow \mathbb{R}$ be a functions satisfying the conditions (A1)-(A4) and $T_{s}^{F}, T_{t}^{F}$ be defined as in Lemma 2.1 with $s, t>0$. Then the following holds:

$$
\left\|T_{s}^{F} x-T_{t}^{F} x\right\|^{2} \leq \frac{s-t}{s}\left\langle T_{s} x-T_{t} x, T_{s} x-x\right\rangle
$$

for all $x \in H$. 
Lemma 2.4 [21] Let $\left\{a_{n}\right\}$ be a sequence in $[0,1]$ such that $\sum_{n=1}^{\infty} a_{n}=1$. Then we have the following:

$$
\left\|\sum_{n=1}^{\infty} a_{n} x_{n}\right\|^{2} \leq \sum_{n=1}^{\infty} a_{n}\left\|x_{n}\right\|^{2}
$$

for any bounded sequence $\left\{x_{n}\right\}$ in a Hilbert space $H$.

Lemma 2.5 (Demiclosedness principle) Let $T$ be a nonexpansive mapping on a closed convex subset $C$ of a real Hilbert space $H$. Then $I-T$ is demiclosed at any point $y \in H$, that is, if $x_{n} \rightarrow x$ and $x_{n}-T x_{n} \rightarrow y \in H$, then $x-T x=y$.

Lemma 2.6 [22] Assume that $\left\{a_{n}\right\}$ is a sequence of nonnegative numbers such that

$$
a_{n+1} \leq\left(1-\gamma_{n}\right) a_{n}+\delta_{n}
$$

for each $n \geq 0$, where $\left\{\gamma_{n}\right\}$ is a sequence in $(0,1)$ and $\left\{\delta_{n}\right\}$ is a sequence in $\mathbb{R}$ such that

(1) $\sum_{n=1}^{\infty} \gamma_{n}=\infty$;

(2) $\lim \sup _{n \rightarrow \infty} \delta_{n} / \gamma_{n} \leq 0$ or $\sum_{=1}^{\infty}\left|\delta_{n}\right|<\infty$.

Then $\lim _{n \rightarrow \infty} a_{n}=0$.

Lemma $2.7[23,24]$ Let $U$ and $V$ be nonexpansive mappings. For $\sigma \in(0,1)$, define $S=$ $\sigma U+(1-\sigma) V$. Suppose that $\operatorname{Fix}(U) \cap \operatorname{Fix}(V) \neq \emptyset$. Then $\operatorname{Fix}(U) \cap \operatorname{Fix}(V)=\operatorname{Fix}(S)$.

From [24] we can see that Lemma 2.7 holds whenever $U$ and $V$ are self or non-self mappings.

Lemma 2.8 [24] Let $C$ be a nonempty closed convex subset of a Hilbert space $H$ and $T$ : $C \rightarrow H$ be a nonexpansive mapping with $\operatorname{Fix}(T) \neq \emptyset$. Let $P_{C}$ be the metric projection from $H$ onto $C$. Then $\operatorname{Fix}\left(P_{C} T\right)=\operatorname{Fix}(T)=\operatorname{Fix}\left(T P_{C}\right)$.

Remark 2.2 Let $S_{1}, S_{2}: C \rightarrow H$ be two nonexpansive mappings with $\operatorname{Fix}\left(S_{1}\right) \cap \operatorname{Fix}\left(S_{2}\right) \neq \emptyset$. Let $\sigma \in(0,1)$ and define the mapping $S: C \rightarrow H$ by $S=\sigma S_{1}+(1-\sigma) S_{2}$. By Lemmas 2.7 and 2.8 , it is easy to see that $\operatorname{Fix}\left(P_{C} S\right)=\operatorname{Fix}\left(P_{C} S_{1}\right) \cap \operatorname{Fix}\left(P_{C} S_{2}\right)$.

From Remark 2.2, we get the following result.

Lemma 2.9 Let $\left\{B_{i}\right\}_{i=1}^{N}$ be a finite family of inverse strongly monotone mappings from $C$ to $H$ with the constants $\left\{\beta_{i}\right\}_{i=1}^{N}$ and assume that $\bigcap_{i=1}^{N} \operatorname{VI}\left(C, B_{i}\right) \neq \emptyset$. Let $B=\sum_{i=1}^{N} \alpha_{i} B_{i}$ with $\left\{\alpha_{i}\right\}_{i=1}^{N} \subset(0,1)$ and $\sum_{i=1}^{N} \alpha_{i}=1$. Then $B: C \rightarrow H$ is a $\beta$-inverse strongly monotone mapping with $\beta=\min \left\{\beta_{1}, \ldots, \beta_{N}\right\}$ and $\operatorname{VI}(C, B)=\bigcap_{i=1}^{N} \operatorname{VI}\left(C, B_{i}\right)$. 
Proof It is easy to show that $B$ is a $\beta$-inverse strongly monotone mapping. In fact, for all $x, y \in C$, by Lemma 2.4 , we have

$$
\begin{aligned}
\beta\|B x-B y\|^{2} & =\beta\left\|\sum_{i=1}^{N} \alpha_{i}\left(B_{i} x-B_{i} y\right)\right\|^{2} \\
& \leq \beta \sum_{i=1}^{N} \alpha_{i}\left\|B_{i} x-B_{i} y\right\|^{2} \\
& \leq \sum_{i=1}^{N} \alpha_{i} \beta_{i}\left\|B_{i} x-B_{i} y\right\|^{2} \\
& \leq \sum_{i=1}^{N} \alpha_{i}\left\langle x-y, B_{i} x-B_{i} y\right\rangle \\
& =\langle x-y, B x-B y\rangle,
\end{aligned}
$$

which implies that $B$ is a $\beta$-inverse strongly monotone mapping.

Next, we prove that $\operatorname{VI}(C, B)=\bigcap_{i=1}^{N} \operatorname{VI}\left(C, B_{i}\right)$. Obviously, we have

$$
\bigcap_{i=1}^{N} \mathrm{VI}\left(C, B_{i}\right) \subset \mathrm{VI}(C, B) .
$$

Now, for any $w \in \operatorname{VI}(C, B)$, we show that $w \in \bigcap_{i=1}^{N} \operatorname{VI}\left(C, B_{i}\right)$. Take a constant $\lambda \in(0,2 \beta]$. Then $I-\lambda B$ is nonexpansive. Note that $I-\lambda B=\sum_{i=1}^{N} \alpha_{i}\left(I-\lambda B_{i}\right)$ and each $I-\lambda B_{i}$ is nonexpansive. From Remark 2.2, it follows that

$$
\operatorname{Fix}\left(P_{C}(I-\lambda B)\right)=\bigcap_{i=1}^{N} \operatorname{Fix}\left(P_{C}\left(I-\lambda B_{i}\right)\right) .
$$

Thus we have

$$
w \in \mathrm{VI}(C, B) \quad \Longleftrightarrow w=P_{C}(I-\lambda B) w=P_{C}\left(I-\lambda B_{i}\right) w \quad \Longleftrightarrow w \in \operatorname{VI}\left(C, B_{i}\right)
$$

for each $i=1, \ldots, N$. Therefore, $w \in \bigcap_{i=1}^{N} \operatorname{VI}\left(C, B_{i}\right)$. This completes the proof.

\section{Main result}

Now, we give the main results of this paper.

Theorem 3.1 Let $H_{1}, H_{2}$ be two real Hilbert spaces and $C \subset H_{1}, Q \subset H_{2}$ be nonempty closed convex subsets. Let $A_{i}: H_{1} \rightarrow H_{2}$ be a bounded linear operator for each $i=1, \ldots, N_{1}$ with $N_{1} \in \mathbb{N}$ and $B_{i}: C \rightarrow H_{1}$ be a $\beta_{i}$-inverse strongly monotone operator for each $i=$ $1, \ldots, N_{2}$ with $N_{2} \in \mathbb{N}$. Assume that $F: C \times C \rightarrow \mathbb{R}$ satisfies (A1)-(A4), $F_{i}: Q \times Q \rightarrow \mathbb{R}$ $\left(i=1, \ldots, N_{1}\right)$ satisfies (A1)-(A4). Let $\left\{S_{n}\right\}$ be a countable family of nonexpansive mappings from $C$ into $C$. Assume that $\Theta=\Gamma \cap \Omega \cap \mathrm{VI} \neq \emptyset$, where $\Gamma=\bigcap_{n=1}^{\infty} \operatorname{Fix}\left(S_{n}\right), \Omega=\{z \in C$ : 
$z \in \mathrm{EP}(F)$ and $\left.A_{i} z \in \operatorname{EP}\left(F_{i}\right), i=1, \ldots, N_{1}\right\}$ and $\mathrm{VI}=\bigcap_{i=1}^{N_{2}} \mathrm{VI}\left(C, B_{i}\right)$. Let $\left\{\gamma_{1}, \ldots, \gamma_{N_{2}}\right\} \subset(0,1)$ with $\sum_{i=1}^{N_{2}} \gamma_{i}=1$. Take $v, x_{1} \in C$ arbitrarily and define an iterative scheme in the following manner:

$$
\left\{\begin{array}{l}
u_{i, n}=T_{r_{n}}^{F}\left(I-\gamma A_{i}^{*}\left(I-T_{r_{n}}^{F_{i}}\right) A_{i}\right) x_{n}, \quad i=1, \ldots, N_{1} \\
y_{n}=P_{C}\left(I-\lambda_{n}\left(\sum_{i=1}^{N_{2}} \gamma_{i} B_{i}\right)\right)\left(\frac{1}{N_{1}} \sum_{i=1}^{N_{1}} u_{i, n}\right) \\
x_{n+1}=\alpha_{n} v+\sum_{i=1}^{n}\left(\alpha_{i-1}-\alpha_{i}\right) S_{i} y_{n}
\end{array}\right.
$$

for each $i=1, \ldots, N_{1}$ and $n \in \mathbb{N}$, where $\left\{r_{n}\right\} \subset(r, \infty)$ with $r>0,\left\{\lambda_{n}\right\} \subset(0,2 \beta)$ with $\beta=$ $\min \left\{\beta_{1}, \ldots, \beta_{N_{2}}\right\}$ and $\gamma \subset\left(0,1 / L^{2}\right], L=\max \left\{L_{1}, \ldots, L_{N_{1}}\right\}$ and $L_{i}$ is the spectral radius of the operator $A_{i}^{*} A_{i}$ and $A_{i}^{*}$ is the adjoint of $A_{i}$ for each $i \in\left\{1, \ldots, N_{1}\right\}$, and $\left\{\alpha_{n}\right\} \subset(0,1)$ is a strictly decreasing sequence. Let $\alpha_{0}=1$ and assume that the control sequences $\left\{\alpha_{n}\right\},\left\{\lambda_{n}\right\}$, $\left\{r_{n}\right\}$ satisfy the following conditions:

(1) $\lim _{n \rightarrow \infty} \alpha_{n}=0$ and $\sum_{n=1}^{\infty} \alpha_{n}=\infty$;

(2) $\sum_{n=1}^{\infty}\left|r_{n+1}-r_{n}\right|<\infty$ and $\sum_{n=1}^{\infty}\left|\lambda_{n+1}-\lambda_{n}\right|<\infty$;

(3) $\lim _{n \rightarrow \infty} \lambda_{n}=\lambda>0$.

Then the sequence $\left\{x_{n}\right\}$ defined by (3.1) converges strongly to a point $z=P_{\Theta} v$.

Proof We first show that, for each $i=1, \ldots, N_{1}$ and $n \in \mathbb{N}, A_{i}^{*}\left(I-T_{r_{n}}^{F_{i}}\right) A_{i}$ is a $\frac{1}{2 L_{i}^{2}}$-inverse strongly monotone mapping. In fact, since $T_{r_{n}}^{F_{i}}$ is (firmly) nonexpansive and $I-T_{r_{n}}^{F_{i}}$ is $\frac{1}{2}$ inverse strongly monotone, we have

$$
\begin{aligned}
& \left\|A_{i}^{*}\left(I-T_{r_{n}}^{F_{i}}\right) A_{i} x-A_{i}^{*}\left(I-T_{r_{n}}^{F_{i}}\right) A_{i} y\right\|^{2} \\
& \quad=\left\langle A_{i}^{*}\left(I-T_{r_{n}}^{F_{i}}\right)\left(A_{i} x-A_{i} y\right), A_{i}^{*}\left(I-T_{r_{n}}^{F_{i}}\right)\left(A_{i} x-A_{i} y\right)\right\rangle \\
& \quad=\left\langle\left(I-T_{r_{n}}\right)\left(A_{i} x-A_{i} y\right), A_{i} A_{i}^{*}\left(I-T_{r_{n}}^{F_{i}}\right)\left(A_{i} x-A_{i} y\right)\right\rangle \\
& \quad \leq L_{i}^{2}\left\langle\left(I-T_{r_{n}}^{F_{i}}\right)\left(A_{i} x-A_{i} y\right),\left(I-T_{r_{n}}^{F_{i}}\right)\left(A_{i} x-A_{i} y\right)\right\rangle \\
& \quad=L_{i}^{2}\left\|\left(I-T_{r_{n}}^{F_{i}}\right)\left(A_{i} x-A_{i} y\right)\right\|^{2} \\
& \quad \leq 2 L_{i}^{2}\left\langle A_{i} x-A_{i} y,\left(I-T_{r_{n}}^{F_{i}}\right)\left(A_{i} x-A_{i} y\right)\right\rangle \\
& \quad=2 L_{i}^{2}\left\langle x-y, A_{i}^{*}\left(I-T_{r_{n}}^{F_{i}}\right) A_{i} x-A_{i}^{*}\left(I-T_{r_{n}}^{F_{i}}\right) A_{i} y\right\rangle
\end{aligned}
$$

for all $x, y \in H_{1}$, which implies that $A_{i}^{*}\left(I-T_{r_{n}}^{F_{i}}\right) A_{i}$ is a $\frac{1}{2 L_{i}^{2}}$-inverse strongly monotone mapping. Note that $\gamma \in\left(0, \frac{1}{L_{i}^{2}}\right]$. Thus $I-\gamma A_{i}^{*}\left(I-T_{r_{n}}^{F_{i}}\right) A_{i}$ is nonexpansive for each $i=1, \ldots, N_{1}$ and $n \in \mathbb{N}$.

Now, we complete the proof by the next steps.

Step $1 .\left\{x_{n}\right\}$ is bounded.

Let $p \in \Theta$. Then $p=T_{r_{n}}^{F_{i}} p$ and $\left(I-\gamma A_{i}^{*}\left(I-T_{r_{n}}^{F_{i}}\right) A_{i}\right) p=p$. Thus we have

$$
\begin{aligned}
\left\|u_{i, n}-p\right\| & =\left\|T_{r_{n}}^{F}\left(I-\gamma A_{i}^{*}\left(I-T_{r_{n}}^{F}\right) A_{i}\right) x_{n}-T_{r_{n}}^{F_{i}}\left(I-\gamma A_{i}^{*}\left(I-T_{r_{n}}^{F_{i}}\right) A_{i}\right) p\right\| \\
& \leq\left\|\left(I-\gamma A_{i}^{*}\left(I-T_{r_{n}}^{F_{i}}\right) A_{i}\right) x_{n}-\left(I-\gamma A^{*}\left(I-T_{r_{n}}^{F_{i}}\right) A_{i}\right) p\right\| \\
& \leq\left\|x_{n}-p\right\| .
\end{aligned}
$$


Let $B=\sum_{i=1}^{N_{2}} \gamma_{i} B_{i}$. Then $B$ is a $\beta$-inverse strongly monotone mapping. Since $\left\{\lambda_{n}\right\} \subset(0,2 \beta)$, $I-\lambda_{n} B$ is nonexpansive. Thus from (3.2), we have

$$
\begin{aligned}
\left\|y_{n}-p\right\| & =\left\|P_{C}\left(I-\lambda_{n} B\right) \frac{1}{N_{1}} \sum_{i=1}^{N_{1}} u_{i, n}-P_{C}\left(I-\lambda_{n} B\right) p\right\| \\
& \leq\left\|\left(I-\lambda_{n} B\right) \frac{1}{N_{1}} \sum_{i=1}^{N_{1}} u_{i, n}-\left(I-\lambda_{n} B\right) p\right\| \\
& \leq\left\|\frac{1}{N_{1}} \sum_{i=1}^{N_{1}} u_{i, n}-p\right\| \\
& \leq \frac{1}{N_{1}} \sum_{i=1}^{N_{1}}\left\|u_{i, n}-p\right\| \\
& \leq\left\|x_{n}-p\right\| .
\end{aligned}
$$

Thus from (3.3), it follows that

$$
\begin{aligned}
\left\|x_{n+1}-p\right\| & =\left\|\alpha_{n}(v-p)+\sum_{i=1}^{n}\left(\alpha_{i-1}-\alpha_{i}\right)\left(S_{i} y_{n}-S_{i} p\right)\right\| \\
& \leq \alpha_{n}\|v-p\|+\sum_{i=1}^{n}\left(\alpha_{i-1}-\alpha_{i}\right)\left\|y_{n}-p\right\| \\
& \leq \alpha_{n}\|v-p\|+\sum_{i=1}^{n}\left(\alpha_{i-1}-\alpha_{i}\right)\left\|x_{n}-p\right\| \\
& =\alpha_{n}\|v-p\|+\left(1-\alpha_{n}\right)\left\|x_{n}-p\right\| \\
& \leq \max \left\{\|v-p\|,\left\|x_{n}-p\right\|\right\}
\end{aligned}
$$

for all $n \in \mathbb{N}$, which implies that $\left\{x_{n}\right\}$ is bounded and so are $\left\{u_{i, n}\right\}\left(i=1, \ldots, N_{1}\right)$ and $\left\{y_{n}\right\}$.

Step 2. $\lim _{n \rightarrow \infty}\left\|x_{n+1}-x_{n}\right\|=0$ and $\lim _{n \rightarrow \infty}\left\|u_{i, n+1}-u_{i, n}\right\|=0$ for each $i=1, \ldots, N_{1}$.

Since the mappings $I-\gamma A^{*}\left(I-T_{r_{n}}^{F_{i}}\right) A$ are nonexpansive, by Lemmas 2.2 and 2.3, we have

$$
\begin{aligned}
\left\|u_{i, n+1}-u_{i, n}\right\| \\
=\left\|T_{r_{n+1}}^{F}\left(I-\gamma A_{i}^{*}\left(I-T_{r_{n+1}}^{F_{i}}\right) A_{i}\right) x_{n+1}-T_{r_{n}}^{F}\left(I-\gamma A_{i}^{*}\left(I-T_{r_{n}}^{F_{i}}\right) A_{i}\right) x_{n}\right\| \\
\leq\left\|\left(I-\gamma A_{i}^{*}\left(I-T_{r_{n+1}}^{F_{i}}\right) A_{i}\right) x_{n+1}-\left(I-\gamma A_{i}^{*}\left(I-T_{r_{n}}^{F_{i}}\right) A_{i}\right) x_{n}\right\| \\
\quad+\frac{\left|r_{n+1}-r_{n}\right|}{r_{n+1}}\left\|T_{r_{n+1}}^{F}\left(I-\gamma A_{i}^{*}\left(I-T_{r_{n+1}}^{F_{i}}\right) A_{i}\right) x_{n+1}-\left(I-\gamma A_{i}^{*}\left(I-T_{r_{n+1}}^{F_{i}}\right) A_{i}\right) x_{n+1}\right\| \\
\leq\left\|x_{n+1}-x_{n}\right\|+\left\|\left(I-\gamma A_{i}^{*}\left(I-T_{r_{n+1}}^{F_{i}}\right) A_{i}\right) x_{n}-\left(I-\gamma A_{i}^{*}\left(I-T_{r_{n}}^{F_{i}}\right) A_{i}\right) x_{n}\right\| \\
\quad+\frac{\left|r_{n+1}-r_{n}\right|}{r_{n+1}} \delta_{n+1} \\
=\left\|x_{n+1}-x_{n}\right\|+\left\|\gamma A_{i}^{*}\left(T_{r_{n+1}}^{F_{i}} A_{i} x_{n}-T_{r_{n}}^{F_{i}} A_{i} x_{n}\right)\right\|+\frac{\left|r_{n+1}-r_{n}\right|}{r_{n+1}} \delta_{n+1}
\end{aligned}
$$




$$
\begin{aligned}
\leq & \left\|x_{n+1}-x_{n}\right\|+\gamma\left\|A_{i}^{*}\right\|\left[\frac{\left|r_{n+1}-r_{n}\right|}{r_{n+1}}\left|\left\langle T_{r_{n+1}}^{F_{i}} A_{i} x_{n}-T_{r_{n}}^{F_{i}} A_{i} x_{n}, T_{r_{n+1}}^{F_{i}} A_{i} x_{n}-A_{i} x_{n}\right\rangle\right|\right]^{\frac{1}{2}} \\
& +\frac{\left|r_{n+1}-r_{n}\right|}{r} \delta_{n+1} \\
\leq & \left\|x_{n+1}-x_{n}\right\|+\gamma\left\|A_{i}^{*}\right\|\left[\frac{\left|r_{n+1}-r_{n}\right|}{r} \sigma_{n+1}\right]^{\frac{1}{2}}+\frac{\left|r_{n+1}-r_{n}\right|}{r} \delta_{n+1} \\
\leq & \left\|x_{n+1}-x_{n}\right\|+\eta_{i, n+1},
\end{aligned}
$$

where

$$
\begin{aligned}
& \sigma_{n+1}=\sup _{n \in \mathbb{N}}\left|\left\langle T_{r_{n+1}}^{F_{i}} A_{i} x_{n}-T_{r_{n}}^{F_{i}} A_{i} x_{n}, T_{r_{n+1}}^{F_{i}} A_{i} x_{n}-A_{i} x_{n}\right\rangle\right|, \\
& \delta_{n+1}=\sup _{n \in \mathbb{N}}\left\|T_{r_{n+1}}^{F}\left(I-\gamma A_{i}^{*}\left(I-T_{r_{n+1}}^{F_{i}}\right) A_{i}\right) x_{n+1}-\left(I-\gamma A_{i}^{*}\left(I-T_{r_{n+1}}^{F_{i}}\right) A_{i}\right) x_{n+1}\right\|,
\end{aligned}
$$

and

$$
\eta_{i, n+1}=\gamma\left\|A_{i}^{*}\right\|\left[\frac{\left|r_{n+1}-r_{n}\right|}{r} \sigma_{n+1}\right]^{\frac{1}{2}}+\frac{\left|r_{n+1}-r_{n}\right|}{r} \delta_{n+1} .
$$

Note that

$$
\begin{aligned}
\| & \left(I-\lambda_{n+1} B\right) \frac{1}{N_{1}} \sum_{i=1}^{N_{1}} u_{i, n+1}-\left(I-\lambda_{n} B\right) \frac{1}{N_{1}} \sum_{i=1}^{N_{1}} u_{i, n} \| \\
= & \left\|\left(I-\lambda_{n+1} B\right) \frac{1}{N_{1}} \sum_{i=1}^{N_{1}} u_{i, n+1}-\left(I-\lambda_{n+1} B\right) \frac{1}{N_{1}} \sum_{i=1}^{N_{1}} u_{i, n}+\left(\lambda_{n}-\lambda_{n+1}\right) B w_{n}\right\| \\
\leq & \left\|\left(I-\lambda_{n+1} B\right) \frac{1}{N_{1}} \sum_{i=1}^{N_{1}} u_{i, n+1}-\left(I-\lambda_{n+1} B\right) \frac{1}{N_{1}} \sum_{i=1}^{N_{1}} u_{i, n}\right\| \\
& +\left|\lambda_{n}-\lambda_{n+1}\right|\left\|B w_{n}\right\| \\
\leq & \frac{1}{N_{1}} \sum_{i=1}^{N_{1}}\left\|u_{i, n+1}-u_{i, n}\right\|+\left|\lambda_{n}-\lambda_{n+1}\right|\left\|B w_{n}\right\|,
\end{aligned}
$$

where $w_{n}=\frac{1}{N_{1}} \sum_{i=1}^{N_{1}} u_{i, n}$. Let $M_{1}=\sup _{n \in \mathbb{N}}\left\|B w_{n}\right\|$. By (3.1), (3.4), and (3.5), we have

$$
\begin{aligned}
\left\|y_{n+1}-y_{n}\right\| & =\left\|P_{C}\left(I-\lambda_{n+1} B\right) \frac{1}{N_{1}} \sum_{i=1}^{N_{1}} u_{i, n+1}-P_{C}\left(I-\lambda_{n} B\right) \frac{1}{N_{1}} \sum_{i=1}^{N_{1}} u_{i, n}\right\| \\
& \leq\left\|\left(I-\lambda_{n+1} B\right) \frac{1}{N_{1}} \sum_{i=1}^{N_{1}} u_{i, n+1}-\left(I-\lambda_{n} B\right) \frac{1}{N_{1}} \sum_{i=1}^{N_{1}} u_{i, n}\right\| \\
& \leq \frac{1}{N_{1}} \sum_{i=1}^{N_{1}}\left\|u_{i, n+1}-u_{i, n}\right\|+\left|\lambda_{n}-\lambda_{n+1}\right|\left\|B w_{n}\right\| \\
& \leq\left\|x_{n+1}-x_{n}\right\|+\frac{1}{N_{1}} \sum_{i=1}^{N_{1}} \eta_{i, n+1}+\left|\lambda_{n}-\lambda_{n+1}\right| M_{1} .
\end{aligned}
$$


Since $\left\{\alpha_{n}\right\}$ is strictly decreasing, by using (3.6), we have

$$
\begin{aligned}
& \left\|x_{n+1}-x_{n}\right\| \\
& =\left\|\left(\alpha_{n}-\alpha_{n-1}\right) v+\sum_{i=1}^{n-1}\left(\alpha_{i-1}-\alpha_{i}\right)\left(S_{i} y_{n}-S_{i} y_{n-1}\right)+\left(\alpha_{n-1}-\alpha_{n}\right) S_{n} y_{n}\right\| \\
& \leq\left(\alpha_{n-1}-\alpha_{n}\right)\|v\|+\sum_{i=1}^{n-1}\left(\alpha_{i-1}-\alpha_{i}\right)\left\|S_{i} y_{n}-S_{i} y_{n-1}\right\|+\left(\alpha_{n-1}-\alpha_{n}\right)\left\|S_{n} y_{n}\right\| \\
& \quad \leq\left(\alpha_{n-1}-\alpha_{n}\right)\|v\|+\sum_{i=1}^{n-1}\left(\alpha_{i-1}-\alpha_{i}\right)\left\|y_{n}-y_{n-1}\right\|+\left(\alpha_{n-1}-\alpha_{n}\right)\left\|S_{n} y_{n}\right\| \\
& \quad=\left(\alpha_{n-1}-\alpha_{n}\right)\|v\|+\left(1-\alpha_{n-1}\right)\left\|y_{n}-y_{n-1}\right\|+\left(\alpha_{n-1}-\alpha_{n}\right)\left\|S_{n} y_{n}\right\| \\
& \quad \leq\left(1-\alpha_{n-1}\right)\left\|x_{n}-x_{n-1}\right\|+\frac{1}{N_{1}} \sum_{i=1}^{N_{1}} \eta_{i, n}+\left|\lambda_{n-1}-\lambda_{n}\right| M_{1}+\left(\alpha_{n-1}-\alpha_{n}\right) M_{2},
\end{aligned}
$$

where $M_{2}=\sup \left\{\left\|S_{n} y_{n}\right\|+\|v\|: n \in \mathbb{N}\right\}$. By (i) and (ii) and Lemma 2.6, we conclude that

$$
\lim _{n \rightarrow \infty}\left\|x_{n+1}-x_{n}\right\|=0
$$

Further, by (3.4) and (3.6), we have

$$
\lim _{n \rightarrow \infty}\left\|y_{n+1}-y_{n}\right\|=0, \quad \lim _{n \rightarrow \infty}\left\|u_{i, n+1}-u_{i, n}\right\|=0, \quad i \in\left\{1, \ldots, N_{1}\right\}
$$

Step 3. $\lim _{n \rightarrow \infty}\left\|S_{i} x_{n}-x_{n}\right\| \rightarrow 0$ for each $i \in \mathbb{N}$.

First, we show that $\lim _{n \rightarrow \infty}\left\|u_{i, n}-x_{n}\right\|=0$ for each $i \in\left\{1, \ldots, N_{1}\right\}$. Since each $A_{i}^{*}\left(I-T_{r_{n}}^{F_{i}}\right) A_{i}$ is $\frac{1}{2 L_{i}^{2}}$-inverse strongly monotone, by (3.1), we have

$$
\begin{aligned}
\left\|u_{i, n}-p\right\|^{2}= & \left\|T_{r_{n}}^{F}\left(I-\gamma A_{i}^{*}\left(I-T_{r_{n}}^{F_{i}}\right) A_{i}\right) x_{n}-T_{r_{n}}^{F}\left(I-\gamma A_{i}^{*}\left(I-T_{r_{n}}^{F_{i}}\right) A_{i}\right) p\right\|^{2} \\
\leq & \left\|\left(I-\gamma A_{i}^{*}\left(I-T_{r_{n}}^{F_{i}}\right) A_{i}\right) x_{n}-\left(I-\gamma A_{i}^{*}\left(I-T_{r_{n}}^{F_{i}}\right) A_{i}\right) p\right\|^{2} \\
= & \left\|\left(x_{n}-p\right)-\gamma\left(A_{i}^{*}\left(I-T_{r_{n}}^{F_{i}}\right) A_{i} x_{n}-A_{i}^{*}\left(I-T_{r_{n}}^{F_{i}}\right) A_{i} p\right)\right\|^{2} \\
= & \left\|x_{n}-p\right\|^{2}-2 \gamma\left(x_{n}-p, A_{i}^{*}\left(I-T_{r_{n}}^{F_{i}}\right) A_{i} x_{n}-A_{i}^{*}\left(I-T_{r_{n}}^{F_{i}}\right) A_{i} p\right\rangle \\
& +\gamma^{2}\left\|A_{i}^{*}\left(I-T_{r_{n}}^{F_{i}}\right) A_{i} x_{n}-A_{i}^{*}\left(I-T_{r_{n}}^{F_{i}}\right) A_{i} p\right\|^{2} \\
\leq & \left\|x_{n}-p\right\|^{2}-\frac{\gamma}{L_{i}^{2}}\left\|A_{i}^{*}\left(I-T_{r_{n}}^{F_{i}}\right) A_{i} x_{n}-A_{i}^{*}\left(I-T_{r_{n}}^{F_{i}}\right) A_{i} p\right\|^{2} \\
& +\gamma^{2}\left\|A_{i}^{*}\left(I-T_{r_{n}}^{F_{i}}\right) A_{i} x_{n}-A_{i}^{*}\left(I-T_{r_{n}}^{F_{i}}\right) A_{i} p\right\|^{2} \\
= & \left\|x_{n}-p\right\|^{2}+\gamma\left(\gamma-\frac{1}{L_{i}^{2}}\right)\left\|A_{i}^{*}\left(I-T_{r_{n}}^{F_{i}}\right) A_{i} x_{n}-A_{i}^{*}\left(I-T_{r_{n}}^{F_{i}}\right) A_{i} p\right\|^{2} \\
= & \left\|x_{n}-p\right\|^{2}+\gamma\left(\gamma-\frac{1}{L_{i}^{2}}\right)\left\|A_{i}^{*}\left(I-T_{r_{n}}^{F_{i}}\right) A_{i} x_{n}\right\|^{2} .
\end{aligned}
$$


From Lemma 2.4 and (3.9), it follows that

$$
\begin{aligned}
\left\|x_{n+1}-p\right\|^{2}= & \left\|\alpha_{n}(v-p)+\sum_{i=1}^{n}\left(\alpha_{i-1}-\alpha_{i}\right)\left(S_{i} y_{n}-p\right)\right\|^{2} \\
\leq & \alpha_{n}\|v-p\|^{2}+\sum_{i=1}^{n}\left(\alpha_{i-1}-\alpha_{i}\right)\left\|S_{i} y_{n}-p\right\|^{2} \\
\leq & \alpha_{n}\|v-p\|^{2}+\sum_{i=1}^{n}\left(\alpha_{i-1}-\alpha_{i}\right)\left\|y_{n}-p\right\|^{2} \\
= & \alpha_{n}\|v-p\|^{2}+\left(1-\alpha_{n}\right)\left\|y_{n}-p\right\|^{2} \\
\leq & \alpha_{n}\|v-p\|^{2}+\left(1-\alpha_{n}\right) \sum_{i=1}^{N_{1}} \frac{1}{N_{1}}\left\|u_{i, n}-p\right\|^{2} \\
\leq & \alpha_{n}\|v-p\|^{2} \\
& +\left(1-\alpha_{n}\right) \sum_{i=1}^{N_{1}} \frac{1}{N_{1}}\left[\left\|x_{n}-p\right\|^{2}+\gamma\left(\gamma-\frac{1}{L_{i}^{2}}\right)\left\|A_{i}^{*}\left(I-T_{r_{n}}^{F_{i}}\right) A_{i} x_{n}\right\|^{2}\right] \\
& +\left(1-\alpha_{n}\right) \sum_{i=1}^{N_{1}} \frac{1}{N_{1}} \gamma\left(\gamma-\frac{1}{L_{i}^{2}}\right)\left\|A_{i}^{*}\left(I-T_{r_{n}}^{F_{i}}\right) A_{i} x_{n}\right\|^{2} . \\
& \alpha_{n}\|v-p\|^{2}+\left(1-\alpha_{n}\right)\left\|x_{n}-p\right\|^{2} \\
& +\left(1-\alpha_{n}\right) \sum_{i=1}^{N_{1}} \frac{1}{N_{1}} \gamma\left(\gamma-\frac{1}{L_{i}^{2}}\right)\left\|A_{i}^{*}\left(I-T_{r_{n}}^{F_{i}}\right) A_{i} x_{n}\right\|^{2} \\
& \left(\|v\|^{2}+\left\|x_{n}-p\right\|^{2}\right. \\
& \\
& (1)
\end{aligned}
$$

Since $\gamma<\frac{1}{L^{2}}=\max \left\{\frac{1}{L_{1}^{2}}, \ldots, \frac{1}{L_{N_{1}}^{2}}\right\}$, we have

$$
\begin{aligned}
(1- & \left.\alpha_{n}\right) \frac{1}{N_{1}} \gamma\left(\frac{1}{L_{i}^{2}}-\gamma\right)\left\|A_{i}^{*}\left(I-T_{r_{n}}^{F_{i}}\right) A_{i} x_{n}\right\|^{2} \\
& \leq\left(1-\alpha_{n}\right) \sum_{i=1}^{N_{1}} \frac{1}{N_{1}} \gamma\left(\frac{1}{L_{i}^{2}}-\gamma\right)\left\|A_{i}^{*}\left(I-T_{r_{n}}^{F_{i}}\right) A_{i} x_{n}\right\|^{2} \\
& \leq \alpha_{n}\|v-p\|^{2}+\left\|x_{n}-p\right\|^{2}-\left\|x_{n+1}-p\right\|^{2} \\
& \leq \alpha_{n}\|v-p\|^{2}+\left\|x_{n}-x_{n+1}\right\|\left(\left\|x_{n}-p\right\|+\left\|x_{n+1}-p\right\|\right) .
\end{aligned}
$$

Since $\alpha_{n} \rightarrow 0$, by (3.7), we have

$$
\lim _{n \rightarrow \infty}\left\|A_{i}^{*}\left(I-T_{r_{n}}^{F_{i}}\right) A_{i} x_{n}\right\|=0
$$

for each $i \in\left\{1, \ldots, N_{1}\right\}$, which implies that

$$
\lim _{n \rightarrow \infty}\left\|\left(I-T_{r_{n}}^{F_{i}}\right) A_{i} x_{n}\right\|=0
$$


for each $i \in\left\{1, \ldots, N_{1}\right\}$. Since $T_{r_{n}}^{F}$ is firmly nonexpansive and $I-\gamma A_{i}^{*}\left(I-T_{r_{n}}^{F_{i}}\right) A_{i}$ is nonexpansive, by (3.1), we have

$$
\begin{aligned}
\left\|u_{i, n}-p\right\|^{2}= & \left\|T_{r_{n}}^{F}\left(x_{n}+\gamma A_{i}^{*}\left(T_{r_{n}}^{F_{i}}-I\right) A_{i} x_{n}\right)-T_{r_{n}}^{F}(p)\right\|^{2} \\
\leq & \left\langle u_{i, n}-p, x_{n}+\gamma A_{i}^{*}\left(T_{r_{n}}^{i}-I\right) A_{i} x_{n}-p\right\rangle \\
= & \frac{1}{2}\left\{\left\|u_{i, n}-p\right\|^{2}+\left\|x_{n}+\gamma A_{i}^{*}\left(T_{r_{n}}^{F_{i}}-I\right) A_{i} x_{n}-p\right\|^{2}\right. \\
& \left.-\left\|u_{i, n}-p-\left[x_{n}+\gamma A_{i}^{*}\left(T_{r_{n}}^{F_{i}}-I\right) A_{i} x_{n}-p\right]\right\|^{2}\right\} \\
= & \frac{1}{2}\left\{\left\|u_{i, n}-p\right\|^{2}+\left\|\left(I-\gamma A_{i}^{*}\left(I-T_{r_{n}}^{F_{i}}\right) A_{i}\right) x_{n}-\left(I-\gamma A_{i}^{*}\left(I-T_{r_{n}}^{F_{2}}\right) A_{i}\right) p\right\|^{2}\right. \\
& \left.-\left\|u_{i, n}-x_{n}-\gamma A_{i}^{*}\left(T_{r_{n}}^{F_{i}}-I\right) A_{i} x_{n}\right\|^{2}\right\} \\
\leq & \frac{1}{2}\left\{\left\|u_{i, n}-p\right\|^{2}+\left\|x_{n}-p\right\|^{2}-\left\|u_{i, n}-x_{n}-\gamma A_{i}^{*}\left(T_{r_{n}}^{F_{i}}-I\right) A_{i} x_{n}\right\|^{2}\right\} \\
= & \frac{1}{2}\left\{\left\|u_{i, n}-p\right\|^{2}+\left\|x_{n}-p\right\|^{2}-\left[\left\|u_{i, n}-x_{n}\right\|^{2}+\gamma^{2}\left\|A_{i}^{*}\left(T_{r_{n}}^{F_{i}}-I\right) A_{i} x_{n}\right\|^{2}\right.\right. \\
& \left.\left.-2 \gamma\left\langle u_{i, n}-x_{n}, A_{i}^{*}\left(T_{r_{n}}^{F_{i}}-I\right) A_{i} x_{n}\right\rangle\right]\right\},
\end{aligned}
$$

which implies that

$$
\left\|u_{i, n}-p\right\|^{2} \leq\left\|x_{n}-p\right\|^{2}-\left\|u_{i, n}-x_{n}\right\|^{2}+2 \gamma\left\|u_{i, n}-x_{n}\right\|\left\|A_{i}^{*}\left(T_{r_{n}}^{F_{i}}-I\right) A_{i} x_{n}\right\| .
$$

Now, from (3.1) and (3.12), it follows that

$$
\begin{aligned}
\left\|x_{n+1}-p\right\|^{2} \leq & \alpha_{n}\|v-p\|^{2}+\left(1-\alpha_{n}\right)\left\|y_{n}-p\right\|^{2} \\
\leq & \alpha_{n}\|v-p\|^{2}+\left(1-\alpha_{n}\right) \sum_{i=1}^{N_{1}} \frac{1}{N_{1}}\left\|u_{i, n}-p\right\|^{2} \\
\leq & \alpha_{n}\|v-p\|^{2}+\left(1-\alpha_{n}\right) \sum_{i=1}^{N_{1}} \frac{1}{N_{1}}\left(\left\|x_{n}-p\right\|^{2}-\left\|u_{i, n}-x_{n}\right\|^{2}\right. \\
& \left.+2 \gamma\left\|u_{i, n}-x_{n}\right\|\left\|A_{i}^{*}\left(T_{r_{n}}^{F_{i}}-I\right) A_{i} x_{n}\right\|\right) \\
\leq & \alpha_{n}\|v-p\|^{2}+\left\|x_{n}-p\right\|^{2}-\left(1-\alpha_{n}\right) \sum_{i=1}^{N_{1}} \frac{1}{N_{1}}\left\|u_{i, n}-x_{n}\right\|^{2} \\
& +2 \gamma \sum_{i=1}^{N_{1}} \frac{1}{N_{1}}\left\|u_{i, n}-x_{n}\right\|\left\|A_{i}^{*}\left(T_{r_{n}}^{F_{i}}-I\right) A_{i} x_{n}\right\|,
\end{aligned}
$$

and so

$$
\begin{aligned}
\left(1-\alpha_{n}\right) \frac{1}{N_{1}}\left\|u_{i, n}-x_{n}\right\|^{2} \leq & \left(1-\alpha_{n}\right) \sum_{i=1}^{N_{1}} \frac{1}{N_{1}}\left\|u_{i, n}-x_{n}\right\|^{2} \\
\leq & \alpha_{n}\|\nu-p\|^{2}+\left\|x_{n}-x_{n+1}\right\|\left(\left\|x_{n}-p\right\|+\left\|x_{n+1}-p\right\|\right) \\
& \left.+2 \gamma \sum_{i=1}^{N_{1}} \frac{1}{N_{1}}\left(\left\|u_{i, n}\right\|+\left\|x_{n}\right\|\right)\left\|A_{i}^{*}\left(T_{r_{n}}^{F_{i}}-I\right) A_{i} x_{n}\right\|\right) .
\end{aligned}
$$


Since $\alpha_{n} \rightarrow 0$, both $\left\{u_{i, n}\right\}$ and $\left\{x_{n}\right\}$ are bounded, by (3.7) and (3.10), we have

$$
\lim _{n \rightarrow \infty}\left\|u_{i, n}-x_{n}\right\|=0
$$

for each $i \in\left\{1, \ldots, N_{1}\right\}$.

Next, we show that $\lim _{n \rightarrow \infty}\left\|y_{n}-u_{n}\right\|=0$, where $u_{n}=\frac{1}{N_{1}} \sum_{i=1}^{N_{1}} u_{i, n}$. Note that $p=$ $P_{C}\left(I-\lambda_{n} B\right) p$. By (3.1), we have

$$
\begin{aligned}
\left\|x_{n+1}-p\right\|^{2} \leq & \alpha_{n}\|v-p\|^{2}+\left(1-\alpha_{n}\right)\left\|y_{n}-p\right\|^{2} \\
\leq & \alpha_{n}\|v-p\|^{2}+\left(1-\alpha_{n}\right)\left\|u_{n}-p-\lambda_{n}\left(B u_{n}-B p\right)\right\|^{2} \\
= & \alpha_{n}\|v-p\|^{2} \\
& +\left(1-\alpha_{n}\right)\left(\left\|u_{n}-p\right\|^{2}-2 \lambda_{n}\left\langle u_{n}-p, B u_{n}-B p\right\rangle+\lambda_{n}^{2}\left\|B u_{n}-B p\right\|^{2}\right) \\
\leq & \alpha_{n}\|v-p\|^{2} \\
& +\left(1-\alpha_{n}\right)\left(\left\|u_{n}-p\right\|^{2}-2 \lambda_{n} \beta\left\|B u_{n}-B p\right\|^{2}+\lambda_{n}^{2}\left\|B u_{n}-B p\right\|^{2}\right) \\
\leq & \alpha_{n}\|v-p\|^{2} \\
& +\left(1-\alpha_{n}\right)\left(\left\|x_{n}-p\right\|^{2}-2 \lambda_{n} \beta\left\|B u_{n}-B p\right\|^{2}+\lambda_{n}^{2}\left\|B u_{n}-B p\right\|^{2}\right) \\
= & \alpha_{n}\|v-p\|^{2}+\left(1-\alpha_{n}\right)\left\|x_{n}-p\right\|^{2} \\
& +\left(1-\alpha_{n}\right) \lambda_{n}\left(\lambda_{n}-2 \beta\right)\left\|B u_{n}-B p\right\|^{2}
\end{aligned}
$$

and so

$$
\begin{aligned}
& \left(1-\alpha_{n}\right) \lambda_{n}\left(2 \beta-\lambda_{n}\right)\left\|B u_{n}-B p\right\|^{2} \\
& \quad \leq \alpha_{n}\|v-p\|^{2}+\left\|x_{n}-x_{n+1}\right\|\left(\left\|x_{n}-p\right\|+\left\|x_{n+1}-p\right\|\right) .
\end{aligned}
$$

Since $\alpha_{n} \rightarrow 0$ and $0<\lim _{n \rightarrow \infty} \lambda_{n}=\lambda<2 \beta$, by (3.7), we have

$$
\lim _{n \rightarrow \infty}\left\|B u_{n}-B p\right\|=0
$$

Since $P_{C}$ is firmly nonexpansive and $\left(I-\lambda_{n} B\right)$ is nonexpansive, by (3.1), we have

$$
\begin{aligned}
\left\|y_{n}-p\right\|^{2}= & \left\|P_{C}\left(u_{n}-\lambda_{n} B u_{n}\right)-P_{C}\left(p-\lambda_{n} B p\right)\right\|^{2} \\
\leq & \left\langle y_{n}-p, u_{n}-\lambda_{n} B u_{n}-\left(p-\lambda_{n} B p\right)\right\rangle \\
= & \frac{1}{2}\left(\left\|y_{n}-p\right\|^{2}+\left\|\left(I-\lambda_{n} B\right) u_{n}-\left(I-\lambda_{n} B\right) p\right\|^{2}-\left\|y_{n}-u_{n}+\lambda_{n}\left(B u_{n}-B p\right)\right\|^{2}\right) \\
\leq & \frac{1}{2}\left(\left\|y_{n}-p\right\|^{2}+\left\|u_{n}-p\right\|^{2}-\left\|y_{n}-u_{n}+\lambda_{n}\left(B u_{n}-B p\right)\right\|^{2}\right) \\
= & \frac{1}{2}\left(\left\|y_{n}-p\right\|^{2}+\left\|u_{n}-p\right\|^{2}-\left\|y_{n}-u_{n}\right\|^{2}-\lambda_{n}^{2}\left\|B u_{n}-B p\right\|^{2}\right. \\
& \left.-2 \lambda_{n}\left\langle y_{n}-u_{n}, B u_{n}-B p\right\rangle\right) \\
\leq & \frac{1}{2}\left(\left\|y_{n}-p\right\|^{2}+\left\|u_{n}-p\right\|^{2}-\left\|y_{n}-u_{n}\right\|^{2}-\lambda_{n}^{2}\left\|B u_{n}-B p\right\|^{2}\right. \\
& \left.+2 \lambda_{n}\left\|y_{n}-u_{n}\right\|\left\|B u_{n}-B p\right\|\right)
\end{aligned}
$$


and so

$$
\begin{aligned}
\left\|y_{n}-p\right\|^{2} \leq & \left\|u_{n}-p\right\|^{2}-\left\|y_{n}-u_{n}\right\|^{2}-\lambda_{n}^{2}\left\|B u_{n}-B p\right\|^{2} \\
& +2 \lambda_{n}\left\|y_{n}-u_{n}\right\|\left\|B u_{n}-B p\right\| \\
\leq & \left\|x_{n}-p\right\|^{2}-\left\|y_{n}-u_{n}\right\|^{2}+2 \lambda_{n}\left\|y_{n}-u_{n}\right\|\left\|B u_{n}-B p\right\| .
\end{aligned}
$$

From (3.1) and (3.15), we have

$$
\begin{aligned}
\left\|x_{n+1}-p\right\|^{2} \leq & \alpha_{n}\|v-p\|^{2}+\left(1-\alpha_{n}\right)\left\|y_{n}-p\right\|^{2} \\
\leq & \alpha_{n}\|v-p\|^{2} \\
& +\left(1-\alpha_{n}\right)\left(\left\|x_{n}-p\right\|^{2}-\left\|y_{n}-u_{n}\right\|^{2}+2 \lambda_{n}\left\|y_{n}-u_{n}\right\|\left\|B u_{n}-B p\right\|\right) \\
\leq & \alpha_{n}\|v-p\|^{2}+\left\|x_{n}-p\right\|^{2}-\left(1-\alpha_{n}\right)\left\|y_{n}-u_{n}\right\|^{2} \\
& +2\left(1-\alpha_{n}\right) \lambda_{n}\left\|y_{n}-u_{n}\right\|\left\|B u_{n}-B p\right\| .
\end{aligned}
$$

Therefore, we have

$$
\begin{aligned}
\left(1-\alpha_{n}\right)\left\|y_{n}-u_{n}\right\|^{2} \leq & \alpha_{n}\|v-p\|^{2}+\left\|x_{n}-x_{n+1}\right\|\left(\left\|x_{n+1}-p\right\|+\left\|x_{n}-p\right\|\right) \\
& +2\left(1-\alpha_{n}\right) \lambda_{n}\left(\left\|y_{n}\right\|+\left\|u_{n}\right\|\right)\left\|B u_{n}-B p\right\| .
\end{aligned}
$$

Since $\lim _{n \rightarrow \infty} \alpha_{n}=0$ and both $\left\{y_{n}\right\}$ and $\left\{u_{n}\right\}$ are bounded, by (3.7) and (3.14), we have

$$
\lim _{n \rightarrow \infty}\left\|y_{n}-u_{n}\right\|=0
$$

Further, from (3.7), (3.13), (3.16), and

$$
\begin{aligned}
\left\|x_{n+1}-y_{n}\right\| & \leq\left\|x_{n+1}-x_{n}\right\|+\left\|x_{n}-u_{n}\right\|+\left\|u_{n}-y_{n}\right\| \\
& \leq\left\|x_{n+1}-x_{n}\right\|+\sum_{i=1}^{N_{1}} \frac{1}{N_{1}}\left\|x_{n}-u_{i, n}\right\|+\left\|u_{n}-y_{n}\right\|,
\end{aligned}
$$

it follows that

$$
\lim _{n \rightarrow \infty}\left\|x_{n+1}-y_{n}\right\|=0
$$

Now, from (3.1), it follows that

$$
\sum_{i=1}^{n}\left(\alpha_{i-1}-\alpha_{i}\right)\left(S_{i} y_{n}-y_{n}\right)=x_{n+1}-y_{n}-\alpha_{n}\left(v-y_{n}\right)
$$

Since $\left\{\alpha_{n}\right\}$ is strictly decreasing, for each $i \in \mathbb{N}$, by (2.1) and (3.18), we have

$$
\begin{aligned}
\left(\alpha_{i-1}-\alpha_{i}\right)\left\|S_{i} y_{n}-y_{n}\right\|^{2} & \leq \sum_{i=1}^{n}\left(\alpha_{i-1}-\alpha_{i}\right)\left\|S_{i} y_{n}-y_{n}\right\|^{2} \\
& \leq 2 \sum_{i=1}^{n}\left(\alpha_{i-1}-\alpha_{i}\right)\left\langle S_{i} y_{n}-y_{n}, p-y_{n}\right\rangle
\end{aligned}
$$




$$
\begin{aligned}
& =2\left\langle x_{n+1}-y_{n}, y_{n}-p\right\rangle-2 \alpha_{n}\left\langle v-y_{n}, p-y_{n}\right\rangle \\
& \leq 2\left\|x_{n+1}-y_{n}\right\|\left\|y_{n}-p\right\|+2 \alpha_{n}\left\|v-y_{n}\right\|\left\|y_{n}-p\right\| .
\end{aligned}
$$

Since $\lim _{n \rightarrow \infty} \alpha_{n}=0$ and $\left\{y_{n}\right\}$ is bounded, by (3.17), one has

$$
\lim _{n \rightarrow \infty}\left\|S_{i} y_{n}-y_{n}\right\|=0
$$

for all $i \in \mathbb{N}$. Further, since

$$
\begin{aligned}
\left\|S_{i} x_{n}-x_{n}\right\| & \leq\left\|S_{i} x_{n}-S_{i} y_{n}\right\|+\left\|S_{i} y_{n}-y_{n}\right\|+\left\|y_{n}-x_{n}\right\| \\
& \leq 2\left\|y_{n}-x_{n}\right\|+\left\|S_{i} y_{n}-y_{n}\right\| \\
& \leq 2\left\|y_{n}-x_{n+1}\right\|+2\left\|x_{n+1}-x_{n}\right\|+\left\|S_{i} y_{n}-y_{n}\right\|,
\end{aligned}
$$

by (3.7), (3.17), and (3.19), we obtain

$$
\lim _{n \rightarrow \infty}\left\|S_{i} x_{n}-x_{n}\right\|=0
$$

for all $i \in \mathbb{N}$.

Step $4 . \lim _{\sup _{n \rightarrow \infty}}\left\langle v-z, x_{n}-z\right\rangle \leq 0$.

Let $z=P_{\Theta} v$. Since $\left\{x_{n}\right\}$ is bounded, we can choose a subsequence $\left\{x_{n_{j}}\right\}$ of $\left\{x_{n}\right\}$ such that

$$
\limsup _{n \rightarrow \infty}\left\langle v-z, x_{n}-z\right\rangle=\lim _{j \rightarrow \infty}\left\langle v-z, x_{n_{j}}-z\right\rangle
$$

Since $\left\{x_{n_{j}}\right\}$ is bounded, there exists a subsequence $\left\{x_{n_{j}}\right\}$ of $\left\{x_{n_{j}}\right\}$ converging weakly to a point $w \in C$. Without loss of generality, we can assume that $x_{n_{j}} \rightarrow w$.

Now, we show that $w \in \Theta$. First of all, we prove that $w \in \Gamma=\bigcap_{i=1}^{\infty} \operatorname{Fix}\left(S_{i}\right)$. In fact, since $x_{n}-S_{i} x_{n} \rightarrow 0$ for each $i \in \mathbb{N}$ and $x_{n_{j}} \rightarrow w$, by Lemma 2.5, we obtain $w \in \bigcap_{i=1}^{\infty} \operatorname{Fix}\left(S_{i}\right)=\Gamma$.

Next, we show that $w \in \Omega$, i.e., $w \in \operatorname{EP}(F)$ and $A_{i} w \in \operatorname{EP}\left(F_{i}\right)$ for each $i=1, \ldots, N_{1}$.

Let $w_{i, n}=\left(I-A_{i}^{*}\left(I-T_{r_{n}}^{F_{i}}\right)\right) A_{i} x_{n}$ for each $i=1, \ldots, N_{1}$. By (3.10) and (3.13) we see that $w_{i, n}-$ $x_{n} \rightarrow 0$ and $T_{r_{n}}^{F} w_{i, n}-w_{i, n} \rightarrow 0$ as $n \rightarrow \infty$. By Lemma 2.2 we see that $\left\|T_{r_{n}}^{F} w_{i, n}-T_{r}^{F} w_{i, n}\right\| \leq$ $\left|1-\frac{r}{r_{n}}\right|\left\|T_{r_{n}}^{F} w_{i, n}-w_{i, n}\right\| \rightarrow 0$ as $n \rightarrow \infty$. Hence $T_{r}^{F} w_{i, n}-w_{i, n} \rightarrow 0$ as $n \rightarrow \infty$ for each $i=$ $1, \ldots, N_{1}$. Since $T_{r}^{F}$ is non-expansive and $\left\{w_{i, n}\right\}$ converges weakly to $w$, by Lemma 2.5 we get $w=T_{r}^{F} w$, i.e., $w \in \operatorname{EP}(F)$. On the other hand, since $\left(I-\gamma A_{i}^{*}\left(I-T_{r_{n}}^{F_{i}}\right) A_{i}\right) x_{n}-x_{n} \rightarrow 0$ (by (3.13)) and $I-\gamma A_{i}^{*}\left(I-T_{r_{n}}^{F_{i}}\right) A_{i}$ is non-expansive, from Lemmas 2.2 and 2.5 it follows that $w=\left(I-\gamma A_{i}^{*}\left(I-T_{r}^{F_{i}}\right) A_{i}\right) w$, i.e., $w=T_{r}^{F} A_{i} w$. Therefore, $w \in \Omega$.

Finally, we prove that $w \in \mathrm{VI}=\bigcap_{i=1}^{N_{2}} \mathrm{VI}\left(C, B_{i}\right)$ by demiclosedness principle. Obviously, we only need to show that $w=P_{C}\left(w-\lambda B_{i} w\right)$, where $\lambda=\lim _{n \rightarrow \infty} \lambda_{n}$. By (3.1) and (3.16), one has $\left\|u_{n}-P_{C}\left(I-\lambda_{n} B\right) u_{n}\right\| \rightarrow 0$, where $u_{n}=\frac{1}{N_{1}} \sum_{i=1}^{N_{1}} u_{i, n}$. Then we have

$$
\begin{aligned}
\left\|u_{n}-P_{C}(I-\lambda B) u_{n}\right\| & \leq\left\|u_{n}-P_{C}\left(I-\lambda_{n} B\right) u_{n}\right\|+\left\|P_{C}\left(I-\lambda_{n} B\right) u_{n}-P_{C}(I-\lambda B) u_{n}\right\| \\
& \leq\left\|u_{n}-P_{C}\left(I-\lambda_{n} B\right) u_{n}\right\|+\left\|\left(I-\lambda_{n} B\right) u_{n}-(I-\lambda B) u_{n}\right\| \\
& \leq\left\|u_{n}-P_{C}\left(I-\lambda_{n} B\right) u_{n}\right\|+\left|\lambda-\lambda_{n}\right|\left\|B u_{n}\right\| .
\end{aligned}
$$

Since $\lambda_{n} \rightarrow \lambda>0,\left\{B u_{n}\right\}$ is bounded and $\left\|u_{n}-P_{C}(I-\lambda B) u_{n}\right\| \rightarrow 0$, we have

$$
\lim _{n \rightarrow \infty}\left\|u_{n}-P_{C}(I-\lambda B) u_{n}\right\|=0
$$


On the other hand, since $\left\{\lambda_{n}\right\} \subset(0,2 \beta)$, one has $\lambda \in(0,2 \beta]$. Thus $I-\lambda B$ is nonexpansive and, further, $P_{C}(I-\lambda B)$ is nonexpansive. Noting that $u_{n_{j}} \rightarrow w$ as $j \rightarrow \infty$, by Lemma 2.5 , we obtain $w=P_{C}(I-\lambda B) w$. By Lemma 2.8, we get $w \in \mathrm{VI}=\bigcap_{i=1}^{N_{2}} \operatorname{VI}\left(C, B_{i}\right)$. Therefore, $w \in \Theta$. By the property on $P_{C}$, we have

$$
\limsup _{n \rightarrow \infty}\left\langle v-z, x_{n}-z\right\rangle=\lim _{j \rightarrow \infty}\left\langle v-z, x_{n_{j}}-z\right\rangle=\langle v-z, w-z\rangle \leq 0
$$

Step 5. $x_{n} \rightarrow z=P_{\Theta} v$ as $n \rightarrow \infty$.

By (3.1), we have

$$
\begin{aligned}
\left\|x_{n+1}-z\right\|^{2} & =\left\|\alpha_{n} v+\sum_{i=1}^{n}\left(\alpha_{i-1}-\alpha_{i}\right) S_{i} y_{n}-z\right\|^{2} \\
& =\alpha_{n}\left\langle v-z, x_{n+1}-z\right\rangle+\sum_{i=1}^{n}\left(\alpha_{i-1}-\alpha_{i}\right)\left\langle S_{i} y_{n}-z, x_{n+1}-z\right\rangle \\
& \leq \alpha_{n}\left\langle v-z, x_{n+1}-z\right\rangle+\frac{\sum_{i=1}^{n}\left(\alpha_{i-1}-\alpha_{i}\right)}{2}\left(\left\|S_{i} y_{n}-z\right\|^{2}+\left\|x_{n+1}-z\right\|^{2}\right) \\
& \leq \alpha_{n}\left\langle v-z, x_{n+1}-z\right\rangle+\frac{\sum_{i=1}^{n}\left(\alpha_{i-1}-\alpha_{i}\right)}{2}\left(\left\|x_{n}-z\right\|^{2}+\left\|x_{n+1}-z\right\|^{2}\right) \\
& =\alpha_{n}\left\langle v-z, x_{n+1}-z\right\rangle+\frac{1-\alpha_{n}}{2}\left(\left\|x_{n}-z\right\|^{2}+\left\|x_{n+1}-z\right\|^{2}\right) \\
& \leq \alpha_{n}\left\langle v-z, x_{n+1}-z\right\rangle+\frac{1-\alpha_{n}}{2}\left\|x_{n}-z\right\|^{2}+\frac{1}{2}\left\|x_{n+1}-z\right\|^{2}
\end{aligned}
$$

which implies that

$$
\left\|x_{n+1}-z\right\|^{2} \leq\left(1-\alpha_{n}\right)\left\|x_{n}-z\right\|^{2}+2 \alpha_{n}\left\langle v-z, x_{n+1}-z\right\rangle
$$

By Lemma 2.6 and (3.21), we can conclude that $\lim _{n \rightarrow \infty}\left\|x_{n}-z\right\|=0$. This completes the proof.

The following results follow directly from Theorem 3.1.

Corollary 3.2 Let $H_{1}, H_{2}$ be two real Hilbert spaces and $C \subset H_{1}, Q \subset H_{2}$ be nonempty closed convex subsets. Let $A: H_{1} \rightarrow H_{2}$ be a bounded linear operator and $B: C \rightarrow H_{1}$ be a $\beta$-inverse strongly monotone operator. Assume that $F: C \times C \rightarrow \mathbb{R}, F_{1}: Q \times Q \rightarrow \mathbb{R}$ are bifunctions satisfying the conditions (A1)-(A4). Let $\left\{S_{n}\right\}$ be countable family of nonexpansive mappings from $C$ into $C$. Assume that $\Theta=\Gamma \cap \Omega \cap \operatorname{VI}(C, B) \neq \emptyset$, where $\Gamma=\bigcap_{n=1}^{\infty} \operatorname{Fix}\left(S_{n}\right)$ and $\Omega=\left\{z \in C: z \in \operatorname{EP}(F)\right.$ and $\left.A z \in \operatorname{EP}\left(F_{1}\right)\right\}$. Take $v \in C$ arbitrarily and define an iterative scheme in the following manner:

$$
\left\{\begin{array}{l}
u_{n}=T_{r_{n}}^{F}\left(I-\gamma A^{*}\left(I-T_{r_{n}}^{F_{1}}\right) A\right) x_{n}, \\
y_{n}=P_{C}\left(u_{n}-\lambda_{n} B u_{n}\right), \\
x_{n+1}=\alpha_{n} v+\sum_{i=1}^{n}\left(\alpha_{i-1}-\alpha_{i}\right) S_{i} y_{n},
\end{array}\right.
$$

for all $n \in \mathbb{N}$, where $\left\{r_{n}\right\} \subset(r, \infty)$ with $r>0,\left\{\lambda_{n}\right\} \subset(0,2 \beta)$, and $\gamma \subset\left(0,1 / L^{2}\right], L$ is the spectral radius of the operator $A^{*} A$ and $A^{*}$ is the adjoint of $A, \alpha_{0}=1$, and $\left\{\alpha_{n}\right\} \subset(0,1)$ is a 
strictly decreasing sequence. Assume that the control sequences $\left\{\alpha_{n}\right\},\left\{\lambda_{n}\right\}$, and $\left\{r_{n}\right\}$ satisfy the following conditions:

(1) $\lim _{n \rightarrow \infty} \alpha_{n}=0$ and $\sum_{n=1}^{\infty} \alpha_{n}=\infty$;

(2) $\sum_{n=1}^{\infty}\left|r_{n+1}-r_{n}\right|<\infty$ and $\sum_{n=1}^{\infty}\left|\lambda_{n+1}-\lambda_{n}\right|<\infty$;

(3) $\lim _{n \rightarrow \infty} \lambda_{n}=\lambda \in(0,2 \beta)$.

Then the sequence $\left\{x_{n}\right\}$ defined by (3.22) converges strongly to a point $z=P_{\Theta} v$.

Corollary 3.3 Let $H_{1}, H_{2}$ be two real Hilbert spaces and $C \subset H_{1}, Q \subset H_{2}$ be nonempty closed convex subsets. Let $A: H_{1} \rightarrow H_{2}$ be a bounded linear operator and $B: C \rightarrow H_{1}$ be a $\beta$-inverse strongly monotone operator. Assume that $F: C \times C \rightarrow \mathbb{R}, F_{1}: Q \times Q \rightarrow \mathbb{R}$ are the bifunctions satisfying the conditions (A1)-(A4). Let $S: C \rightarrow C$ be a nonexpansive mapping. Assume that $\Theta=\operatorname{Fix}(S) \cap \Omega \cap \operatorname{VI}(C, B) \neq \emptyset$, where $\Omega=\left\{z \in C: z \in \operatorname{EP}(F)\right.$ and $\left.A z \in \operatorname{EP}\left(F_{1}\right)\right\}$. Take $v \in C$ arbitrarily and define an iterative scheme in the following manner:

$$
\left\{\begin{array}{l}
u_{n}=T_{r_{n}}^{F}\left(I-\gamma A^{*}\left(I-T_{r_{n}}^{F_{1}}\right) A\right) x_{n}, \\
y_{n}=P_{C}\left(u_{n}-\lambda_{n} B u_{n}\right), \\
x_{n+1}=\alpha_{n} v+\left(1-\alpha_{n}\right) S y_{n}
\end{array}\right.
$$

for all $n \in \mathbb{N}$, where $\left\{r_{n}\right\} \subset(r, \infty)$ with $r>0,\left\{\lambda_{n}\right\} \subset(0,2 \beta)$, and $\gamma \subset\left(0,1 / L^{2}\right], L$ is the spectral radius of the operator $A^{*} A$ and $A^{*}$ is the adjoint of $A,\left\{\alpha_{n}\right\} \subset(0,1)$ is a sequence. Assume that the control sequences $\left\{\alpha_{n}\right\},\left\{\lambda_{n}\right\}$, and $\left\{r_{n}\right\}$ satisfy the following conditions:

(1) $\lim _{n \rightarrow \infty} \alpha_{n}=0$ and $\sum_{n=1}^{\infty} \alpha_{n}=\infty$;

(2) $\sum_{n=1}^{\infty}\left|r_{n+1}-r_{n}\right|<\infty$ and $\sum_{n=1}^{\infty}\left|\lambda_{n+1}-\lambda_{n}\right|<\infty$;

(3) $\lim _{n \rightarrow \infty} \lambda_{n}=\lambda \in(0,2 \beta)$.

Then the sequence $\left\{x_{n}\right\}$ defined by (3.23) converges strongly to a point $z=P_{\Theta} v$.

Remark 3.4 Theorem 3.1 and Corollary 3.3 extend the corresponding one of Kazmi and Rizvi [14] from a nonexpansive mapping to a finite of family of nonexpansive mappings and from a split equilibrium problem to a finite of family of split equilibrium problems. It is a little simple to prove that $w \in \mathrm{VI}$ by the demiclosedness principle in Theorem 3.1.

We give an example to illustrate Theorem 3.1 as follows.

Example 3.5 Let $H_{1}=\mathbb{R}$ and $H_{2}=\mathbb{R}^{2}, C=[0,1]$, and $Q=[0,1] \times[0,1]$. Let $A_{1}: H_{1} \rightarrow H_{2}$ and $A_{2}: H_{1} \rightarrow H_{2}$ defined by $A_{1} x=(x, x)^{T}$ and $A_{2} x=\left(\frac{x}{2}, \frac{x}{2}\right)^{T}$ for each $x \in H_{1}$. Then $A_{1}^{*} y=$ $y_{1}+y_{2}$ and $A_{2}^{*} y=\frac{y_{1}+y_{2}}{2}$ for each $y=\left(y_{1}, y_{2}\right)^{T} \in H_{2}$. Then $L_{1}=2$ and $L_{2}=\frac{1}{2}$, where $L_{1}$ and $L_{2}$ are the spectral radius of $A_{1}^{*} A_{1}$ and $A_{2}^{*} A_{2}$, respectively.

Let $B_{1}=2(x-1)$ and $B_{2}=-4$ for all $x \in C$. Then it is easy to see that $B_{1}$ and $B_{2}$ are $\frac{1}{2}$ and 1inverse strongly monotone operators from $C$ into $H_{1}$. Find that $\mathrm{VI}=\mathrm{VI}\left(C, B_{1}\right) \cap \mathrm{VI}\left(C, B_{2}\right)=$ $\{1\}$. For each $n \in \mathbb{N}$, let $S_{n}: C \rightarrow C$ defined by $S_{n}(x)=x+\frac{1}{3 n}$ for each $x \in\left[0, \frac{1}{2}\right]$ and $S_{n}(x)=x$ for each $x \in\left(\frac{1}{2}, 1\right]$. Then $\left\{S_{n}\right\}$ is a countable family of nonexpansive mappings from $C$ into $C$ and it is easy to see that $\Gamma=\bigcap_{n=1}^{\infty} \operatorname{Fix}\left(S_{n}\right)=\left(\frac{1}{2}, 1\right]$. For each $x, y \in C$, define the bifunction $F: C \times C \rightarrow \mathbb{R}$ by $F(x, y)=x-y$ for all $x, y \in C$. For each $u=\left(u_{1}, u_{2}\right)^{T}$ and $v=\left(v_{1}, v_{2}\right)^{T} \in Q$, define $F_{1}: Q \times Q \rightarrow \mathbb{R}$ and $F_{2}: Q \times Q \rightarrow \mathbb{R}$ by

$$
F_{1}(u, v)=u_{1}+u_{2}-v_{1}-v_{2}
$$


and

$$
F_{2}(u, v)= \begin{cases}0, & \text { if } u=v, \\ 2, & \text { if } u=(1,1) \text { or }\left(\frac{1}{2}, \frac{1}{2}\right) \text { and } v \neq(1,1) \text { or }\left(\frac{1}{2}, \frac{1}{2}\right), \\ -2, & \text { if } v=(1,1) \text { or }\left(\frac{1}{2}, \frac{1}{2}\right) \text { and } u \neq(1,1) \text { or }\left(\frac{1}{2}, \frac{1}{2}\right) \\ u_{1}^{2}+u_{2}^{2}-v_{1}-v_{2}, & \text { otherwise. }\end{cases}
$$

It is easy to check that the bifunctions $F, F_{1}$, and $F_{2}$ satisfy the conditions (A1)-(A4) and $F_{1}$. Moreover, $\Omega=\{1\}$, where $\Omega=\left\{z \in C: z \in \operatorname{EP}(F), A_{1} z \in \operatorname{EP}\left(F_{1}\right)\right.$ and $\left.A_{2} z \in \operatorname{EP}\left(F_{2}\right)\right\}$. Therefore, $\Theta=\Gamma \cap \operatorname{VI} \cap \Omega=\{1\}$.

Let $\alpha_{0}=1, \gamma_{1}=\gamma_{2}=\frac{1}{2}$, and $\gamma=\frac{1}{4}$. For each $n \in \mathbb{N}$, let $r_{n}=2, \lambda_{n}=\frac{1}{4}, \alpha_{n}=\frac{1}{n}$. Then the sequences $\left\{\alpha_{n}\right\},\left\{\lambda_{n}\right\},\left\{r_{n}\right\}$ satisfy the conditions (1)-(3) in Theorem 3.1.

For each $x \in C$ and each $n \in \mathbb{N}$, we compute $T_{r_{n}}^{F_{1}} A_{1} x$, i.e., $T_{r_{n}}^{F_{1}}(x, x)$. Find $z=(1,1)$ such that

$$
\begin{aligned}
F_{1}(z, y)+\frac{1}{r_{n}}\left\langle y-z, z-A_{1} x\right\rangle & =2-\left(y_{1}+y_{2}\right)+\frac{1}{2}\left[\left(y_{1}-1\right)(1-x)+\left(y_{2}-1\right)(1-x)\right] \\
& =2-\left(y_{1}+y_{2}\right)+\frac{1}{2}(1-x)\left(y_{1}+y_{2}-2\right) \\
& =\left[2-\left(y_{1}+y_{2}\right)\right]\left[1-\frac{1}{2}(1-x)\right] \\
& \geq 0
\end{aligned}
$$

for all $y=\left(y_{1}, y_{2}\right) \in Q$. Thus, from Lemma 2.1(1), it follows that $T_{r_{n}}^{F_{1}} A_{1} x=(1,1)$ for each $x \in C$. Similarly, for each $x \in[0,1]$, we can find $z=(1,1)$ such that, for $y=\left(\frac{1}{2}, \frac{1}{2}\right)$,

$$
F_{2}(z, y)+\frac{1}{r_{n}}\left\langle y-z, z-A_{2} x\right\rangle=1-\frac{1}{2}(1-x)=\frac{1}{2}+\frac{x}{4} \geq 0
$$

for $y=(1,1)$,

$$
F_{2}(z, y)+\frac{1}{r_{n}}\left\langle y-z, z-A_{2} x\right\rangle=0
$$

for $y \in Q \backslash\left\{(1,1),\left(\frac{1}{2}, \frac{1}{2}\right)\right\}$,

$$
F_{2}(z, y)+\frac{1}{r_{n}}\left\langle y-z, z-A_{2} x\right\rangle=2+\frac{1}{2}\left[\left(y_{1}-1\right)\left(1-\frac{x}{2}\right)+\left(y_{2}-1\right)\left(1-\frac{x}{2}\right)\right] \geq 0 .
$$

Thus $z=(1,1)=T_{r_{n}}^{F_{2}} A_{2} x$ for all $x \in C$ by Lemma 2.1(1).

Now, take $v=\frac{1}{2}$ and $x_{1}=\frac{1}{4}$ and define the sequence $\left\{x_{n}\right\}$ defined by (3.1). Since each $x_{n} \in C$, from the statement above we get $T_{r_{n}}^{F_{i}} A_{i} x_{n}=(1,1)$ for each $i=1,2$. Furthermore, we 
can get

$$
\begin{aligned}
\left(I-\gamma A_{1}^{*}\left(I-T_{r_{n}}^{F_{1}}\right) A_{1}\right) x_{n} & =\left(x_{n}-\gamma A_{1}^{*}\left(A_{1} x_{n}-T_{r_{n}}^{F_{1}} A_{1} x_{n}\right)\right) \\
& =\left(x_{n}-\gamma A_{1}^{*}\left(\left(x_{n}, x_{n}\right)-(1,1)\right)\right) \\
& =x_{n}-2 \gamma\left(x_{n}-1\right) \\
& =\frac{1+x_{n}}{2} .
\end{aligned}
$$

Note that

$$
\begin{aligned}
F(1, y)+\frac{1}{r_{n}}\left\langle y-z, z-\frac{1+x_{n}}{2}\right\rangle & =1-y+\frac{1}{2}(y-1)\left(1-\frac{1+x_{n}}{2}\right) \\
& =(1-y)\left(1-\frac{1}{2}\left(1-\frac{1+x_{n}}{2}\right)\right) \\
& =(1-y)\left(\frac{1}{2}+\frac{1+x_{n}}{4}\right) \\
& \geq 0
\end{aligned}
$$

for all $y \in C$. Thus $u_{1, n}=1$ by Lemma 2.1(1) for each $n \in \mathbb{N}$. Similarly, we can conclude that $u_{2, n}=1$ for each $n \in \mathbb{N}$.

Next, we compute the sequence $\left\{y_{n}\right\}$. By the definition of $\left\{y_{n}\right\}$, we see that

$$
\begin{aligned}
y_{n} & =P_{C}\left[\left(I-\lambda_{n} \frac{B_{1}+B_{2}}{2}\right) \frac{u_{1, n}+u_{2, n}}{2}\right] \\
& =P_{C}\left(1+\frac{2}{4}\right)=1
\end{aligned}
$$

for all $n \in \mathbb{N}$.

Finally, we compute the sequence $\left\{x_{n}\right\}$ by the following iteration:

$$
\begin{aligned}
x_{n+1} & =\alpha_{n} v+\sum_{i=1}^{n}\left(\alpha_{i-1}-\alpha_{i}\right) S_{i} y_{n} \\
& =\alpha_{n} v+1-\alpha_{n} \\
& =1-\frac{1}{2 n} \\
& \rightarrow 1=P_{\Theta} v=P_{\{1\}} \frac{1}{2}
\end{aligned}
$$

as $n \rightarrow \infty$ as shown by Theorem 3.1.

\section{Applications}

In this section, let $H_{1}, H_{2}$ be two real Hilbert spaces and $C, Q$ be two nonempty closed convex subsets of $H_{1}$ and $H_{2}$, respectively. Let $f: C \rightarrow \mathbb{R}, g: Q \rightarrow \mathbb{R}$ be two operators and $A: H_{1} \rightarrow H_{2}$ be a bounded linear operator. 
We consider the following optimization problem:

$$
\begin{aligned}
& \text { find } x^{*} \in C \text { such that } f\left(x^{*}\right) \leq f(x), \quad \forall x \in C, \\
& \qquad \text { and } y^{*}=A x^{*} \text { such that } g\left(y^{*}\right) \leq g(y), \quad \forall y \in Q .
\end{aligned}
$$

We denote the set of solutions of (4.1) by $\Theta$ and assume that $\Theta \neq \emptyset$. Let $F(x, y)=f(y)-f(x)$ for all $x, y \in C$ and $F_{1}(x, y)=g(y)-g(x)$ for all $x, y \in Q$. Then $F(x, y)$ and $G(x, y)$ satisfy the conditions (A1)-(A4) in Section 2 provided that $f$ is convex and lower semicontinuous on $C$ and $g$ is convex and lower semicontinuous on $Q$. Let $\Omega=\{z \in C: z \in \operatorname{EP}(F)$ and $A z \in$ $\left.\operatorname{EP}\left(F_{1}\right)\right\}$. Obviously, $\Theta=\Omega$.

By Corollary 3.3 with $B=I$ and $S=I$, we have the following iterative algorithm, which strongly converges to a point $z=P_{\Theta} v$, which solves the optimization problem (4.1):

$$
\left\{\begin{array}{l}
u_{n}=T_{r_{n}}^{F}\left(I-\gamma A^{*}\left(I-T_{r_{n}}^{F_{1}}\right) A\right) x_{n}, \\
y_{n}=P_{C}\left(u_{n}-\lambda_{n} u_{n}\right), \\
x_{n+1}=\alpha_{n} v+\left(1-\alpha_{n}\right) y_{n},
\end{array}\right.
$$

where $\left\{r_{n}\right\} \subset(r, \infty)$ with $r>0,\left\{\lambda_{n}\right\} \subset(0,2)$, and $\gamma \subset\left(0,1 / L^{2}\right], L$ is the spectral radius of the operator $A^{*} A$ and $A^{*}$ is the adjoint of $A,\left\{\alpha_{n}\right\} \subset(0,1)$ is a sequence. Assume that the control sequences $\left\{\alpha_{n}\right\},\left\{\lambda_{n}\right\}$, and $\left\{r_{n}\right\}$ satisfy the following conditions:

(1) $\lim _{n \rightarrow \infty} \alpha_{n}=0$ and $\sum_{n=1}^{\infty} \alpha_{n}=\infty$;

(2) $\sum_{n=1}^{\infty}\left|r_{n+1}-r_{n}\right|<\infty, \sum_{n=1}^{\infty}\left|\alpha_{n+1}-\alpha_{n}\right|<\infty$, and $\sum_{n=1}^{\infty}\left|\lambda_{n+1}-\lambda_{n}\right|<\infty$;

(3) $\lim _{n \rightarrow \infty} \lambda_{n}=\lambda \in(0,2)$.

For the special case with $H_{1}=H_{2}$ and $C=Q$, we consider the following multi-objective optimization problem:

$$
\left\{\begin{array}{l}
\min \{f(x), g(x)\} \\
x \in C .
\end{array}\right.
$$

We denote the set of solution of (4.3) by $\Gamma$ and assume that $\Gamma \neq \emptyset$. In (4.2), setting $A=I$ we get the following algorithm, which strongly converges to the solution of multi-objective optimization problem (4.3):

$$
\left\{\begin{array}{l}
u_{n}=T_{r_{n}}^{F}\left(I-\gamma\left(I-T_{r_{n}}^{F_{1}}\right)\right) x_{n}, \\
y_{n}=P_{C}\left(u_{n}-\lambda_{n} u_{n}\right), \\
x_{n+1}=\alpha_{n} v+\left(1-\alpha_{n}\right) y_{n},
\end{array}\right.
$$

where $\gamma \subset\left(0,1 / L^{2}\right], L$ is the spectral radius of the operator $I^{*} I$ and $I^{*}$ is the adjoint of $I$, other parameters such as $\left\{\alpha_{n}\right\},\left\{\lambda_{n}\right\}$, and $\left\{r_{n}\right\}$ satisfy the same conditions (1)-(3).

\section{Conclusion}

In this paper, we construct an iterative algorithm to find a common element of the set of solutions of a finite family of split equilibrium problems and the set of common fixed points of a countable family of nonexpansive mappings in Hilbert spaces. In the proof methods, we use the inverse strong monotonicity of each $A^{*}\left(I-T_{r_{n}}\right) A$, which is such that 
the proof is simple and is different from the ones given in [14-16]. Also, in the results of this paper, we do not assume that each $F_{i}$ is upper semi-continuous in the first argument for each $i=1, \ldots, N_{1}$, which is required in the result in [14-16]. As an application, we solve an optimization problem by the result of this paper.

\section{Competing interests}

The authors declare that they have no competing interests.

\section{Authors' contributions}

All authors read and approved the final manuscript.

\section{Author details}

${ }^{1}$ Department of Mathematics and Physics, North China Electric Power University, Baoding, 071003, China. ${ }^{2}$ Department of Mathematics and Sciences, Shijiazhuang University of Economics, Shijiazhuang, 050031, China. ${ }^{3}$ Department of Mathematics, King Abdulaziz University, Jeddah, 21589, Saudi Arabia. ${ }^{4}$ Department of Mathematics Education and RINS, Gyeongsang National University, Jinju, 660701, Korea. ${ }^{5}$ Center for General Education, China Medical University, Taichung, 40402, Taiwan.

\section{Acknowledgements}

This work is supported by the Natural Science Funds of Hebei (Grant Number: A2015502021), the Fundamental Research Funds for the Central Universities (Grant Number: 2014ZD44) and the Project-sponsored by SRF for ROCS, SEM. The fourth author thanks the Basic Science Research Program through the National Research Foundation of Korea (NRF) funded by the Ministry of Science, ICT and future Planning (Grant Number: 2014R1A2A2A01002100).

Received: 7 July 2015 Accepted: 29 November 2015 Published online: 07 January 2016

\section{References}

1. Chang, SS, Lee, HWJ, Chan, CK: A new method for solving equilibrium problem fixed point problem and variational inequality problem with application to optimization. Nonlinear Anal. 70, 3307-3319 (2009)

2. Katchang, P, Kumam, P: A new iterative algorithm of solution for equilibrium problems, variational inequalities and fixed point problems in a Hilbert space. J. Appl. Math. Comput. 32, 19-38 (2010)

3. Plubtieng, S, Punpaeng, R: A general iterative method for equilibrium problems and fixed point problems in Hilbert spaces. J. Math. Anal. Appl. 336, 455-469 (2007)

4. Qin, X, Shang, M, Su, Y: A general iterative method for equilibrium problems and fixed point problems in Hilbert spaces. Nonlinear Anal. 69, 3897-3909 (2008)

5. Combettes, PL, Hirstoaga, SA: Equilibrium programming using proximal like algorithms. Math. Program. 78, 29-41 (1997)

6. Agarwal, RP, Chen, JW, Cho, YJ: Strong convergence theorems for equilibrium problems and weak Bregman relatively nonexpansive mappings in Banach spaces. J. Inequal. Appl. 2013, 119 (2013)

7. Tada, A, Takahashi, W: Weak and strong convergence theorems for a nonexpansive mapping and an equilibrium problem. J. Optim. Theory Appl. 133, 359-370 (2007)

8. Takahashi, S, Takahashi, W: Viscosity approximation methods for equilibrium problems and fixed point problems in Hilbert spaces. J. Math. Anal. Appl. 331, 506-515 (2007)

9. Takahashi, S, Takahashi, W: Strong convergence theorem for a generalized equilibrium problem and a nonexpansive mapping in a Hilbert space. Nonlinear Anal. 69, 1025-1033 (2008)

10. Bnouhachem, A, Chen, Y: An iterative method for a common solution of generalized mixed equilibrium problems, variational inequalities, and hierarchical fixed point problems. Fixed Point Theory Appl. 2014, 155 (2014)

11. Bnouhachem, $A$ : An interactive method for system of generalized equilibrium problem and fixed point problem. Fixed Point Theory Appl. 2014, 235 (2014)

12. Censor, Y, Gibali, A, Reich, S: Algorithms for the split variational inequality problem. Numer. Algorithms 69, 301-323 (2012)

13. Moudafi, A: Split monotone variational inclusions. J. Optim. Theory Appl. 150, 275-283 (2011)

14. Kazmi, KR, Rizvi, SH: Iterative approximation of a common solution of a split equilibrium problem, a variational inequality problem and a fixed point problem. J. Egypt. Math. Soc. 21, 44-51 (2013)

15. Bnouhachem, A: Algorithms of common solutions for a variational inequality, a split equilibrium problem and a hierarchical fixed point problem. Fixed Point Theory Appl. 2013, 278 (2013)

16. Bnouhachem, A: Strong convergence algorithm for split equilibrium problems and hierarchical fixed point problems Sci. World J. 2014, 390956 (2014)

17. liduka, $\mathrm{H}$, Takahashi, W: Strong convergence theorems for nonexpansive mappings and inverse-strongly monotone mappings. Nonlinear Anal. 61, 341-350 (2005)

18. Takahashi, W: Nonlinear Functional Analysis. Yokohama Publishers, Yokohama (2000)

19. Combettes, PL, Hirstoaga, SA: Equilibrium programming in Hilbert spaces. J. Nonlinear Convex Anal. 6, 117-136 (2005)

20. Cianciaruso, F, Marino, G, Muglia, L, Yao, Y: A hybrid projection algorithm for finding solutions of mixed equilibrium problem and variational inequality problem. Fixed Point Theory Appl. 2010, 383740 (2010)

21. Yang, L, Zhao, F, Kim, JK: Hybrid projection method for generalized mixed equilibrium problem and fixed point problem of infinite family of asymptotically quasi- $\psi$-nonexpansive mappings in Banach spaces. Appl. Math. Comput. $218,6072-6082(2012)$

22. Xu, HK: An iterative approach to quadratic optimization. J. Optim. Theory Appl. 116, 659-678 (2003) 
23. Bruch, RE Jr.: Properties of fixed point sets of nonexpansive mappings in Banach spaces. Trans. Am. Math. Soc. 179, 251-289 (1973)

24. Zhou, HY, Wang, PY, Zhou, Y: Minimum-norm fixed point of nonexpansive mappings with applications. Optimization 64, 799-814 (2015)

Submit your manuscript to a SpringerOpen ${ }^{\circ}$ journal and benefit from:

- Convenient online submission

- Rigorous peer review

- Immediate publication on acceptance

- Open access: articles freely available online

- High visibility within the field

- Retaining the copyright to your article

Submit your next manuscript at $>$ springeropen.com 